Research Article

\title{
More on $\mathscr{D} \alpha$-Closed Sets in Topological Spaces
}

\author{
Xiao-Yan Gao ${ }^{1}$ and Ahmed Mostafa Khalil $\mathbb{D}^{2}$ \\ ${ }^{1}$ School of Mathematics and Statistics, Yulin University, Yulin 719000, China \\ ${ }^{2}$ Department of Mathematics, Faculty of Science, Al-Azhar University, Assiut 71524, Egypt
}

Correspondence should be addressed to Ahmed Mostafa Khalil; a.khalil@azhar.edu.eg

Received 23 February 2021; Revised 1 April 2021; Accepted 8 April 2021; Published 26 April 2021

Academic Editor: Sami Ullah Khan

Copyright (c) 2021 Xiao-Yan Gao and Ahmed Mostafa Khalil. This is an open access article distributed under the Creative Commons Attribution License, which permits unrestricted use, distribution, and reproduction in any medium, provided the original work is properly cited.

\begin{abstract}
The aim of this paper is to present and study topological properties of $\mathscr{D} \alpha$-derived, $\mathscr{D} \alpha$-border, $\mathscr{D} \alpha$-frontier, and $\mathscr{D} \alpha$-exterior of a set based on the concept of $\mathscr{D} \alpha$-open sets. Then, we introduce new separation axioms (i.e., $\mathscr{D} \alpha-R_{0}$ and $\mathscr{D} \alpha-R_{1}$ ) by using the notions of $\mathscr{D} \alpha$-open set and $\mathscr{D} \alpha$-closure. The space of $\mathscr{D} \alpha-R_{0}$ (resp., $\mathscr{D} \alpha-R_{1}$ ) is strictly between the spaces of $\alpha-R_{0}$ (resp., $\alpha-R_{1}$ ) and $g-R_{0}$ (resp., $g-R_{1}$ ). Further, we present the notions of $\mathscr{D} \alpha$-kernel and $\mathscr{D} \alpha$-convergent to a point and discuss the characterizations of interesting properties between $\mathscr{D} \alpha$-closure and $\mathscr{D} \alpha$-kernel. Finally, several properties of weakly $\mathscr{D} \alpha-R_{0}$ space are investigated.
\end{abstract}

\section{Introduction and Preliminaries}

Many researchers (see [1-9]) were interested in general topology-like family (e.g., the family of all $\alpha$-open sets) and also the notion of generalized closed (briefly, g-closed) subset of a topological space [10-14]. In 1982, Dunham [14] used the generalized closed sets to define a novel closure operator and consequently a novel topology $\tau^{*}$, on the space, and discussed several of the properties of this novel topology. Sayed and Khalil [15] introduced and studied a novel type of sets called $\mathscr{D} \alpha$-open sets in topological spaces and studied the notions of $\mathscr{D} \alpha$-continuous, $\mathscr{D} \alpha$-open, and $\mathscr{D} \alpha$-closed functions between topological spaces. Further, they investigated several properties of $\mathscr{D} \alpha$-closed and strongly $\mathscr{D} \alpha$-closed graphs. In fact, research on spaces analogous to topological spaces and generalized closed sets among topological spaces may have certain driving effect on research on theory of rough set, soft set, spatial reasoning, implicational spaces and knowledge spaces, and logic (see [16-18]). For this reason, we will define the notions of $\mathscr{D} \alpha$-derived, $\mathscr{D} \alpha$-border, $\mathscr{D} \alpha$-frontier, and $\mathscr{D} \alpha$-exterior of a set based on the notion of $\mathscr{D} \alpha$-open sets. We will also discuss new separation axioms $\left(\mathscr{D} \alpha-R_{0}\right.$ and $\left.\mathscr{D} \alpha-R_{1}\right)$ by using the notions of $\mathscr{D} \alpha$-open set and $\mathscr{D} \alpha$-closure operator.
The rest of this article is arranged as follows. In this section, we briefly recall several notions: $\alpha$-open set, an $\alpha$-closed set, generalized open set, generalized closed set, $\alpha-$ $R_{0}$ space, $g-R_{0}$ space, $\alpha-R_{1}$ space, $g-R_{0}$ space, $\alpha$-derived, $\alpha$-border, $\alpha$-frontier, and $\alpha$-exterior of a set, which are used in the sequel. In Section 2, we define the notions of $\mathscr{D} \alpha$-derived, $\mathscr{D} \alpha$-border, $\mathscr{D} \alpha$-frontier, and $\mathscr{D} \alpha$-exterior of a set based on $\mathscr{D} \alpha$-open sets. In Section 3, we present the notions $\mathscr{D} \alpha-R_{0}, \mathscr{D} \alpha-R_{1}, \mathscr{D} \alpha$-kernel, and $\mathscr{D} \alpha$-convergent to a point and introduce the characterizations of interesting properties between $\mathscr{D} \alpha$-closure and $\mathscr{D} \alpha$-kernel. In Section 4, we define the weakly $\mathscr{D} \alpha-R_{0}$ space and investigate some properties of weakly $\mathscr{D} \alpha-R_{0}$ space.

Throughout the present paper, two subsets $A$ of a space $(X, \tau), \mathscr{C}(A)$ and $\mathscr{I}(A)$, denote the closure and the interior of $A$, respectively. Since we require the following known definitions, notations, and some properties, we recall in this section.

Definition 1. Let $(X, \tau)$ be a topological space and $A \subseteq X$. Then,

(1) $A$ is $\alpha$-open [1] if $A \subseteq \mathscr{I} \mathscr{C} \mathscr{I}(A)$ and $\alpha$-closed [1] if $\mathscr{C}(\mathscr{I}(\mathscr{C}(A))) \subseteq A$ 
(2) $A$ is generalized closed (briefly, $g$-closed) [10] if $\mathscr{C}(A) \subseteq U$ whenever $A \subseteq U$ and $U$ is open in $X$

(3) $A$ is generalized open (briefly, $g$-open) [10] if $X \backslash A$ is $g$-closed

(4) $A$ is $\mathscr{D} \alpha$-open [15] if $A \subseteq \mathscr{J}^{*} \mathscr{C} \mathscr{J}^{*}(A)$ and $D \alpha$-closed [15] if $\mathscr{C}^{*}\left(\mathscr{I}\left(\mathscr{C}^{*}(A)\right)\right) \subseteq A$

The $\alpha$-closure of a subset $A$ of $X$ [2] is the intersection of all $\alpha$-closed sets containing $A$ and is denoted by $\mathscr{C}_{\alpha}(A)$. The $\alpha$-interior of a subset $A$ of $X$ [2] is the union of all $\alpha$-open sets contained in $A$ and is denoted by $\mathscr{I}_{\alpha}(A)$. The intersection of all $g$-closed sets containing $A$ [14] is called the $g$-closure of $A$ and is denoted by $\mathscr{C}^{*}(A)$ and the $g$-interior of $A$ [19] is the union of all $g$-open sets contained in $A$ and is denoted by $\mathscr{I}^{*}(A)$. The intersection of all $\mathscr{D} \alpha$-closed sets containing $A[15]$ is called the $\mathscr{D} \alpha$-closure of $A$ and is denoted by $\mathscr{C}_{\alpha}^{\mathscr{D}}(A)$ and the $\mathscr{D} \alpha$-interior of $A[15]$ is the union of all $\mathscr{D} \alpha$-open sets contained in $A$ and is denoted by $\mathscr{I}_{\alpha}^{\mathscr{D}}(A)$.

We need the following notations:

(i ) $\alpha O(X)$ (resp., $\alpha \mathscr{C}(X)$ ) denotes the family of all $\alpha$-open sets (resp., $\alpha$-closed sets) in $(X, \tau)$

(ii) $G O(X)$ (resp., $G \mathscr{C}(X)$ ) denotes the family of all generalized open sets (resp., generalized closed sets) in $(X, \tau)$

(iii) $\mathscr{D} \alpha O(X)$ (resp., $\mathscr{D} \alpha \mathscr{C}(X))$ denotes the family of all $\mathscr{D} \alpha$-open sets (resp., $\mathscr{D} \alpha$-closed sets) in $(X, \tau)$

(iv) $\alpha O(X, x)=\{U \mid x \in U \in \alpha O(X, \tau)\}, \quad O(X, x)=$ $\{U \mid x \in U \in \tau\}, \quad$ and $\quad \alpha \mathscr{C}(X, x)=$ $\{U \mid x \in U \in \alpha \mathscr{C}(X, \tau)\}$

(v) $\mathscr{D} \alpha O(X, x)=\{U \mid x \in U \in \mathscr{D} \alpha O(X, \tau)\}$ $\mathscr{D} \alpha \mathscr{C}(X, x)=\{U \mid x \in U \in \mathscr{D} \alpha \mathscr{C}(X, \tau)\}$

and

Definition 2. A topological space $(X, \tau)$ is said to be

(1) $\alpha-R_{0}$ space [20] (resp., $g-R_{0}$ space [21]) if every $\alpha$-open (resp., $g$-open) set contains the $\alpha$-closure (resp., $g$-closure) of each of its singletons

(2) $\alpha-R_{1}$ space [20] (resp., $g-R_{1}$ space [21]) if, for $x, y$ in $X$ with $\mathscr{C}_{\alpha}(\{x\}) \neq \mathscr{C}_{\alpha}(\{y\}) \quad$ (resp., $\left.\mathscr{C}^{*}(\{x\}) \neq \mathscr{C}^{*}(\{y\})\right)$, there exist disjoint $\alpha$-open (resp., $g$-open) sets $U$ and $V$ such that $\mathscr{C}_{\alpha}(\{x\})$ (resp., $\left.\mathscr{C}^{*}(\{x\})\right)$ is a subset of $U$ and $\mathscr{C}_{\alpha}(\{y\})$ (resp., $\left.\mathscr{C}^{*}(\{y\})\right)$ is a subset of $V$

Definition 3 (see [22]). A point $x \in X$ is said to be $\alpha$-limit point of $A$ in topological space $(X, \tau)$ if, for each $\alpha$-open set $U$ containing $x, U \cap(A \backslash\{x\}) \neq \phi$. The set of all $\alpha$-limit points of $A$ is called an $\alpha$-derived set of $A$.

Definition 4 (see [22]). Let $A$ be a subset of a space $X$ :

(1) An $\alpha$-border of $A$ is defined by $b_{\alpha}(A)=A \backslash \mathscr{I}_{\alpha}(A)$

(2) An $\alpha$-frontier of $A$ is defined by $F_{\alpha}(A)=\mathscr{C}_{\alpha}(A) \backslash \mathscr{I}_{\alpha}(A)$
(3) An $\alpha$-exterior of $A$ is defined by $\operatorname{Ext}_{\alpha}(A)=$ $\mathscr{I}_{\alpha}(X \backslash A)$

\section{A $\mathscr{D} \alpha$-Derived, $\mathscr{D} \alpha$-Border, $\mathscr{D} \alpha$-Frontier, and $\mathscr{D} \alpha$-Exterior of a Set}

Definition 5. Let $A$ be a subset of a space $X$. A point $x \in X$ is said to be $\mathscr{D} \alpha$-limit point of $A$ if it satisfies the following assertion:

$$
\forall U \in \mathscr{D} \alpha O(X)(x \in U \Rightarrow U \cap(A \backslash\{x\}) \neq \phi)
$$

The set of all $\mathscr{D} \alpha$-limit points of $A$ is called a $\mathscr{D} \alpha$-derived set of $A$ and is denoted by $d_{\alpha}^{\mathscr{D}}(A)$.

Note that, for a subset $A$ of $X$, a point $x \in X$ is not a $\mathscr{D} \alpha$-limit point of $A$ if and only if there exists a $\mathscr{D} \alpha$-open set $U$ in $X$ such that

$$
x \in U, U \cap(A \backslash\{x\})=\phi,
$$

or, equivalently,

$$
x \in U, U \cap A=\phi, U \cap A=\{x\},
$$

or equivalently,

$$
x \in U, U \cap A \subseteq\{x\}
$$

Theorem 1. Let $A$ and $B$ be subsets of a topological space $X$. Then, the following results hold:

(1) $d_{\alpha}^{\mathscr{D}}(A) \subseteq d_{\alpha}(A)$, where $d_{\alpha}(A)$ is the $\alpha$-derived set ([22], Definition 2.1) of $A$

(2) If $A \subseteq B$, then $d_{\alpha}^{\mathscr{D}}(A) \subseteq d_{\alpha}^{\mathscr{D}}(B)$

(3) $d_{\alpha}^{\mathscr{D}}(A) \cup d_{\alpha}^{\mathscr{D}}(B) \subseteq d_{\alpha}^{\mathscr{D}}(A \cup B)$ and $d_{\alpha}^{\mathscr{D}}(A \cap B) \subseteq d_{\alpha}^{\mathscr{D}}$ $(A) \cap d_{\alpha}^{\mathscr{D}}(B)$

(4) $d_{\alpha}^{\mathscr{D}}\left(d_{\alpha}^{\mathscr{D}}(A)\right) \backslash A \subseteq d_{\alpha}^{\mathscr{D}}(A)$

(5) $d_{\alpha}^{\mathscr{D}}\left(A \cup d_{\alpha}^{\mathscr{D}}(A)\right) \subseteq A \cup d_{\alpha}^{\mathscr{D}}(A)$

Proof. (1) It follows from ([15], Theorem 3.6 (i)).

(2) Let $x \in d_{\alpha}^{\mathscr{D}}(A)$ and $U \in \mathscr{D} \alpha O(X)$ with $x \in U$. Then $(U \cap A) \backslash\{x\} \neq \phi$. Since $A \subseteq B$, it follows that $(U \cap B) \backslash\{x\} \neq \phi$. Therefore $x \in d_{\alpha}^{\mathscr{D}}(B)$.

(3) It follows from (2) above.

(4) Let $x \in d_{\alpha}^{\mathscr{D}}\left(d_{\alpha}^{\mathscr{D}}(A)\right) \backslash A$ and $U \in \mathscr{D} \alpha O(X)$ with $x \in U$. Then $U \cap\left(d_{\alpha}^{D}(A) \backslash\{x\}\right) \neq \phi$. Let $y \in U \cap\left(d_{\alpha}^{\mathscr{D}}(A) \backslash\{x\}\right)$. Then $y \in U$ and $y \in d_{\alpha}^{\mathscr{D}}(A)$, and so $U \in(A \backslash\{y\}) \neq \phi$. If we take $z \in U \cap(A \backslash\{y\})$, then $z \neq x$ for $z \in A$ and $x \notin A$. Hence, $U \in(A \backslash\{y\}) \neq \phi$. Therefore $x \in d_{\alpha}^{\mathscr{D}}(A)$.

(5) Let $x \in d_{\alpha}^{\mathscr{D}}\left(A \cup d_{\alpha}^{\mathscr{D}}(A)\right)$. If $x \in A$, the result is obvious. Suppose that $x \notin A$. Then $U \cap\left(\left(A \cup d_{\alpha}^{\mathscr{D}}(A)\right) \backslash\{x\}\right) \neq \phi$ for all $U \in \mathscr{D} \alpha O(X)$ with $x \in U$. Hence, $(U \cap A) \backslash\{x\} \neq \phi$ or $U \cap\left(d_{\alpha}^{\mathscr{D}}(A) \backslash\{x\}\right) \neq \phi$. The first case implies that $x \in d_{\alpha}^{\mathscr{D}}(A)$. If $U \cap\left(d_{\alpha}^{\mathscr{D}}(A) \backslash\{x\}\right) \neq \phi$, then $x \in d_{\alpha}^{\mathscr{D}}\left(d_{\alpha}^{\mathscr{D}}(A)\right)$. Since $x \notin A$, it follows similarly 
from (4) that $x \in d_{\alpha}^{\mathscr{D}}\left(d_{\alpha}^{\mathscr{D}}(A)\right) \backslash A \subseteq d_{\alpha}^{\mathscr{D}}(A)$. Therefore, $d_{\alpha}^{\mathscr{D}}\left(A \cup d_{\alpha}^{\mathscr{D}}(A)\right) \subseteq A \cup d_{\alpha}^{\mathscr{D}}(A)$ holds.

Theorem 2. Let $A$ be a subset of a topological space $X$. Then $\mathscr{C}_{\alpha}^{\mathscr{D}}(A)=A \cup d_{\alpha}^{\mathscr{D}}(A)$.

Proof. Let $x \in \mathscr{C}_{\alpha}^{\mathscr{D}}(A)$. If $x \in A$, then the proof is complete. If $x \notin A$ and $U \in \mathscr{D} \alpha O(X)$ with $x \in U$, then $(U \cap A) \backslash\{x\} \neq \phi$, and so $x \in d_{\alpha}^{D}(A)$. Hence, $\mathscr{C}_{\alpha}^{\mathscr{D}}(A) \subseteq A \cup d_{\alpha}^{\mathscr{D}}(A)$. The converse follows from ([15], Theorem 2.14 (i)) and $d_{\alpha}^{\mathscr{D}}(A) \subseteq \mathscr{C}_{\alpha}^{\mathscr{D}}(A)$. Thus, $A \cup d_{\alpha}^{\mathscr{D}}(A) \subseteq \mathscr{C}_{\alpha}^{\mathscr{D}}(A)$. Therefore $\mathscr{C}_{\alpha}^{\mathscr{D}}(A)=A \cup d_{\alpha}^{\mathscr{D}}(A)$.

Corollary 1. A subset $A$ is a $\mathscr{D} \alpha$-closed set if and only if it contains the set of the $\mathscr{D} \alpha$-limit points

Theorem 3. Let $A$ and $B$ be subsets of $X$. If $A$ is $D \alpha$-closed, then $\mathscr{C}_{\alpha}^{\mathscr{D}}(A \cap B) \subseteq A \cap \mathscr{C}_{\alpha}^{\mathscr{D}}(B)$.

Proof. It follows from Theorems 2.13 and 2.14 (vi) in [15].

Lemma 1. Let $A$ be a subset of a topological space $X$. If $A$ is $\mathscr{D} \alpha$-closed set, then $d_{\alpha}^{\mathscr{D}}(A) \subseteq A$.

Proof. Suppose that $A$ is a $\mathscr{D} \alpha$-closed set. Let $x \notin A$; that is, $x \in X \backslash A$. Since it is a $\mathscr{D} \alpha$-open, $x$ is not a $\mathscr{D} \alpha$-limit point of $A$, that is, $x \notin d_{\alpha}^{\mathscr{D}}(A)$, because $(X \backslash A) \cap(A \backslash\{x\})=\phi$. Hence, $d_{\alpha}^{\mathscr{D}}(A) \subseteq A$.

Theorem 4. Let $A$ be a subset of a topological space $X$. If $F$ is a $\mathscr{D} \alpha$-closed set of $A$, then $d_{\alpha}^{\mathscr{D}}(A) \subseteq F$.

Proof. By Theorem 1 (2) and Lemma 1, $A \subseteq F$ implies that $d_{\alpha}^{\mathscr{D}}(A) \subseteq d_{\alpha}^{\mathscr{D}}(F) \subseteq F$.

Theorem 5. Let $A$ be a subset of a topological space X. If a point $x \in X$ is a $\mathscr{D} \alpha$-limit point of $A$, then $x$ is also a $\mathscr{D} \alpha$-limit point of $A \backslash\{x\}$.

Proof. The proof is obvious.

Definition 6. Let $A$ be a subset of a topological space $X$. The $\mathscr{D} \alpha$-border of $A$, denoted by $b_{\alpha}^{\mathscr{D}}(A)$, is defined as $b_{\alpha}^{\mathscr{D}}(A)=A \backslash \mathscr{I}_{\alpha}^{\mathscr{D}}(A)$.

Theorem 6. Let $A$ be a subset of a topological space X. Then, the following results hold:

(1) $b_{\alpha}^{\mathscr{D}}(A) \subseteq b_{\alpha}(A)$, where $b_{\alpha}(A)$ is the $\alpha$-border ([22], Definition 2.8) of $A$

(2) $A=\mathscr{I}_{\alpha}^{\mathscr{D}}(A) \cup b_{\alpha}^{\mathscr{D}}(A)$

(3) $\mathscr{I}_{\alpha}^{\mathscr{D}}(A) \cap b_{\alpha}^{\mathscr{D}}(A)=\phi$

(4) $A$ is a $\mathscr{D} \alpha$-open set if and only if $b_{\alpha}^{\mathscr{D}}(A)=\phi$

(5) $b_{\alpha}^{\mathscr{D}}\left(\mathscr{I}_{\alpha}^{\mathscr{D}}(A)\right)=\phi$

(6) $b_{\alpha}^{\mathscr{D}}(A)=A \cap \mathscr{C}_{\alpha}^{\mathscr{D}}(X \backslash A)$

(7) $b_{\alpha}^{\mathscr{D}}(A)=A \cap d_{\alpha}^{\mathscr{D}}(X \backslash A)$
Proof. (1) Since $\mathscr{I}_{\alpha}(A) \subseteq \mathscr{I}_{\alpha}^{\mathscr{D}}(A)$ ([1], Theorem 3.15 (i)), we have

$$
b_{\alpha}^{\mathscr{D}}(A)=A \backslash \mathcal{I}_{\alpha}^{\mathscr{D}}(A) \subseteq A \backslash \mathscr{I}_{\alpha}(A)=b_{\alpha}(A),
$$

(2) and (3) are obvious.

(4) Itfollows from Theorems 3.14 and 3.15 (i) in [15].

(5) Since $\mathscr{I}_{\alpha}^{\mathscr{D}}(A)$ is a $\mathscr{D} \alpha$-open, it follows from (4) that $b_{\alpha}^{\mathscr{D}}\left(\mathscr{I}_{\alpha}^{\mathscr{D}}(A)\right)=\phi$.

(6) Using ([15], Lemma 3.13 (i)), we have

$$
\begin{aligned}
b_{\alpha}^{\mathscr{D}}(A) & =A \backslash \mathscr{J}_{\alpha}^{\mathscr{D}}(A)=A \backslash\left(X \backslash \mathscr{C}_{\alpha}^{\mathscr{D}}(X \backslash A)\right) \\
& =A \cap \mathscr{C}_{\alpha}^{\mathscr{D}}(X \backslash A) .
\end{aligned}
$$

(7) Applying (6) and Theorem 3, we have

$$
\begin{aligned}
b_{\alpha}^{\mathscr{D}}(A) & =A \cap \mathscr{C}_{\alpha}^{\mathscr{D}}(X \backslash A)=A \cap\left((X \backslash A) \cup d_{\alpha}^{\mathscr{D}}(X \backslash A)\right) \\
& =A \cap d_{\alpha}^{\mathscr{D}}(X \backslash A) .
\end{aligned}
$$

The converse of (1) of Theorem 6 is not true in general as shown in the following example.

Example 1. Let $(X, \tau)$ be a topological space, where $X=$ $\{a, b, c\}$ and $\tau=\{\phi,\{a\}, X\} . \quad$ Then, $F_{X}=\{\phi, \quad\{b, c\}$, $X\}, \alpha O(X)=\{\phi,\{a\},\{a, b\},\{a, c\}, X\}, \alpha \mathscr{C}(X)=\{\phi, \quad\{b\}, \quad\{c\}$, $\{b, c\}, X\}, \mathscr{D} \alpha O(X)=\mathscr{D} \alpha \mathscr{C}(X)=P(X)$. Let $A=\{c\}$. Then $b_{\alpha}(A)=\{c\} \nsubseteq b_{\alpha}^{\mathscr{D}}(A)=\phi$.

Definition 7. Let $A$ be a subset of a topological space $X$. The $\mathscr{D} \alpha$-frontier of $A$, denoted by $\operatorname{Fr}_{\alpha}^{\mathscr{D}}(A)$, is defined as $\operatorname{Fr}_{\alpha}^{\mathscr{D}}(A)=\mathscr{C}_{\alpha}^{\mathscr{D}}(A) \backslash \mathscr{J}_{\alpha}^{\mathscr{D}}(A)$.

Lemma 2. Let $A$ be a subset of $X$. If $A$ is a $\mathscr{D} \alpha$-closed subset of $X$, then $b_{\alpha}^{\mathscr{D}}(A)=\operatorname{Fr}_{\alpha}^{\mathscr{D}}(A)$.

Proof. It follows from ([15], Theorem 2.13).

Theorem 7. Let $A$ be a subset of a topological space X. Then the following results hold:

(1) $\operatorname{Fr}_{\alpha}^{\mathscr{D}}(A) \subseteq \operatorname{Fr}_{\alpha}(A)$, where $\operatorname{Fr}_{\alpha}(A)$ is the $\alpha$-frontier ([22], Definition 2.11) of $A$.

(2) $\mathscr{C}_{\alpha}^{\mathscr{D}}(A)=\mathscr{I}_{\alpha}^{\mathscr{D}}(A) \cup \operatorname{Fr}_{\alpha}^{\mathscr{D}}(A)$.

(3) $\mathscr{I}_{\alpha}^{\mathscr{D}}(A) \cap \operatorname{Fr}_{\alpha}^{\mathscr{D}}(A)=\phi$.

(4) $b_{\alpha}^{\mathscr{D}}(A) \subseteq \operatorname{Fr}_{\alpha}^{\mathscr{D}}(A)$.

(5) $\operatorname{Fr}_{\alpha}^{\mathscr{D}}(A)=b_{\alpha}^{\mathscr{D}}(A) \cup\left(d_{\alpha}^{\mathscr{D}}(A) \backslash \mathcal{I}_{\alpha}^{\mathscr{D}}(A)\right)$.

(6) If $A$ is a $\mathscr{D} \alpha$-open set, then $\operatorname{Fr}_{\alpha}^{\mathscr{D}}(A)=b_{\alpha}^{\mathscr{D}}(X \backslash A)$.

(7) $\operatorname{Fr}_{\alpha}^{\mathscr{D}}(A)=\mathscr{C}_{\alpha}^{\mathscr{D}}(A) \cap \mathscr{C}_{\alpha}^{\mathscr{D}}(X \backslash A)$.

(8) $\operatorname{Fr}_{\alpha}^{D}(A)=\operatorname{Fr}_{\alpha}^{D}(X \backslash A)$.

(9) $\operatorname{Fr}_{\alpha}^{\mathscr{D}}(A)$ is a $\mathscr{D} \alpha$-closed set.

(10) $\operatorname{Fr}_{\alpha}^{\mathscr{D}}\left(\operatorname{Fr}_{\alpha}^{\mathscr{D}}(A)\right) \subseteq \operatorname{Fr}_{\alpha}^{\mathscr{D}}(A)$. 
(11) $\operatorname{Fr}_{\alpha}^{\mathscr{D}}\left(\mathscr{I}_{\alpha}^{\mathscr{D}}(A)\right) \subseteq \operatorname{Fr}_{\alpha}^{\mathscr{D}}(A)$.

(12) $\operatorname{Fr}_{\alpha}^{\mathscr{D}}\left(\mathscr{C}_{\alpha}^{\mathscr{D}}(A)\right) \subseteq \operatorname{Fr}_{\alpha}^{\mathscr{D}}(A)$.

(13) $\mathscr{I}_{\alpha}^{\mathscr{D}}(A)=A \backslash F r_{\alpha}^{\mathscr{D}}(A)$.

(14) $\operatorname{Fr}_{\alpha}^{\mathscr{D}}(A)=\mathscr{C}_{\alpha}^{\mathscr{D}}(A) \backslash \mathscr{I}_{\alpha}^{\mathscr{D}}(A)$.

(15) $\mathscr{C}_{\alpha}^{\mathscr{D}}(A) \subseteq A \cup \operatorname{Fr}_{\alpha}^{\mathscr{D}}(A)$.

(16) $X \backslash \operatorname{Fr}_{\alpha}^{\mathscr{D}}(A)=\mathscr{I}_{\alpha}^{\mathscr{D}}(A) \cup \mathscr{I}_{\alpha}^{\mathscr{D}}(X \backslash A)$.

\section{Proof}

(1) Since $\mathscr{C}_{\alpha}^{\mathscr{D}}(A) \subseteq \mathscr{C}_{\alpha}(A)$ ([15], Theorem 2.14 (i)) and $\mathscr{I}_{\alpha}(A) \subseteq \mathscr{I}_{\alpha}^{\mathscr{D}}(A)$ ([15], Theorem 3.15 (i)), we have $\operatorname{Fr}_{\alpha}^{\mathscr{D}}(A)=\mathscr{C}_{\alpha}^{\mathscr{D}}(A) \backslash \mathscr{J}_{\alpha}^{\mathscr{D}}(A) \subseteq$ $\mathscr{C}_{\alpha}(A) \backslash \mathscr{I}_{\alpha}(A)=\operatorname{Fr}_{\alpha}(A)$.

(2) It is obvious.

(3) $\mathscr{I}_{\alpha}^{\mathscr{D}}(A) \cap \operatorname{Fr}_{\alpha}^{\mathscr{D}}(A)=\left(\mathscr{C}_{\alpha}^{\mathscr{D}}(A) \backslash \mathscr{I}_{\alpha}^{\mathscr{D}}(A)\right)=\phi$.

(4) Since $A \subseteq \mathscr{C}_{\alpha}^{\mathscr{D}}(A)$ ([15], Theorem 2.14 (i)), we have $b_{\alpha}^{\mathscr{D}}(A)=A \backslash \mathscr{I}_{\alpha}^{\mathscr{D}}(A) \subseteq \mathscr{C}_{\alpha}^{\mathscr{D}}(A) \backslash \mathscr{I}_{\alpha}^{\mathscr{D}}(A)=\operatorname{Fr}_{\alpha}(A)$.

(5) Using Theorem 2, we have

$$
\begin{aligned}
\operatorname{Fr}_{\alpha}^{\mathscr{D}}(A) & =\mathscr{C}_{\alpha}^{\mathscr{D}}(A) \backslash \mathcal{I}_{\alpha}^{\mathscr{D}}(A) \\
& =\left(A \cup d_{\alpha}^{\mathscr{D}}(A)\right) \cap\left(X \backslash \mathcal{I}_{\alpha}^{\mathscr{D}}(A)\right) \\
& =\left(A \backslash \mathcal{I}_{\alpha}^{\mathscr{D}}(A)\right) \cup\left(d_{\alpha}^{\mathscr{D}}(A) \backslash \mathcal{F}_{\alpha}^{\mathscr{D}}(A)\right) \\
& =b_{\alpha}^{\mathscr{D}}(A) \cup\left(d_{\alpha}^{\mathscr{D}}(A) \backslash \mathcal{I}_{\alpha}^{\mathscr{D}}(A)\right) .
\end{aligned}
$$

(6) It follows from (5) above, Theorem 6 (4), (7), and ([15], Theorem 3.14).

(7) It follows from ([15], Lemma 3.13 (ii)).

(8) It follows from (7) above.

(9) $\mathscr{C}_{\alpha}^{\mathscr{D}}\left(\operatorname{Fr}_{\alpha}^{\mathscr{D}}(A)\right)=\mathscr{C}_{\alpha}^{\mathscr{D}}\left(\mathscr{C}_{\alpha}^{\mathscr{D}}(A) \cap \mathscr{C}_{\alpha}^{\mathscr{D}}(X \backslash A)\right) \subseteq \quad \mathscr{C}_{\alpha}^{\mathscr{D}}$ $\left(\mathscr{C}_{\alpha}^{\mathscr{D}}(A)\right) \cap \mathscr{C}_{\alpha}^{\mathscr{D}}\left(\mathscr{C}_{\alpha}^{\mathscr{D}}(X \backslash A)\right)=\mathscr{C}_{\alpha}^{\mathscr{D}}(A) \cap \mathscr{C}_{\alpha}^{\mathscr{D}}(X \backslash$ $A)=\operatorname{Fr}_{\alpha}^{D}(A)$

Obviously, $\operatorname{Fr}_{\alpha}^{\mathscr{D}}(A) \subseteq \mathscr{C}_{\alpha}^{\mathscr{D}}\left(\operatorname{Fr}_{\alpha}^{\mathscr{D}}(A)\right)$ ([15], Theorem $2.14(\mathrm{i}))$, and so $\operatorname{Fr}_{\alpha}^{\mathscr{D}}(A)=\mathscr{C}_{\alpha}^{\mathscr{D}}\left(\operatorname{Fr}_{\alpha}^{\mathscr{D}}(A)\right)$. Hence, $\operatorname{Fr}_{\alpha}^{\mathscr{D}}(A)$ is a $\mathscr{D} \alpha$-closed set.

(10) It follows from (9) above and Lemma 2.

(11) It follows from Definition 7 and ([15], Theorem 3.15 (vi)).

(12) It follows from Definition 7 and ([15], Theorem 2.14 (vi)).

(13) $A \backslash \operatorname{Fr}_{\alpha}^{\mathscr{D}}=A \backslash\left(\mathscr{C}_{\alpha}^{\mathscr{D}}(A) \backslash \mathscr{I}_{\alpha}^{\mathscr{D}}(A)\right)=A \cap\left(\left(X \backslash \quad \mathscr{C}_{\alpha}^{\mathscr{D}}\right.\right.$ $\left.(A)) \cup \mathscr{I}_{\alpha}^{\mathscr{D}}(A)\right)=\phi \cup\left(A \cup \mathscr{I}_{\alpha}^{\mathscr{D}}(A)\right)=\mathscr{I}_{\alpha}^{\mathscr{D}}(A)$

(14) It follows from (7) above and ([15], Lemma 3.13 (ii)).

(15) $A \cup \operatorname{Fr}_{\alpha}^{\mathscr{D}}(A)=A \cup\left(\mathscr{C}_{\alpha} \quad \mathscr{D}(A) \cap \quad \mathscr{C}_{\alpha}^{\mathscr{D}}(X \backslash A)\right)=$ $\left(A \cup \mathscr{C}_{\alpha}^{\mathscr{D}}(A)\right) \cap\left(A \cup \mathscr{C}_{\alpha}^{\mathscr{D}}(X \backslash A)\right)=\mathscr{C}_{\alpha}^{\mathscr{D}}(A) \cap(A \cup$ $\left.\mathscr{C}_{\alpha}^{\mathscr{D}}(X \backslash A)\right) \supseteq \mathscr{C}_{\alpha}^{\mathscr{D}}(A) \cap(A \cup(X \backslash A))=\mathscr{C}_{\alpha}^{\mathscr{D}}(A) \cap$ $X=\mathscr{C}_{\alpha}^{\mathscr{D}}(A)$

(16) $\mathscr{I}_{\alpha}^{\mathscr{D}}(A) \cup \mathscr{I}_{\alpha}^{\mathscr{D}}(X \backslash A)=\left(X \backslash\left(X \backslash \mathscr{G}_{\alpha}^{\mathscr{D}}(A)\right)\right) \cup(X \backslash$ $\left.\left(X \backslash \mathscr{J}_{\alpha}^{\mathscr{D}}(X \backslash A)\right)\right)=X \backslash\left(\left(X \backslash \mathscr{G}_{\alpha}^{\mathscr{D}}(A)^{\alpha}\right) \cap \mathscr{I}_{\alpha}^{\mathscr{D}}(X \backslash\right.$

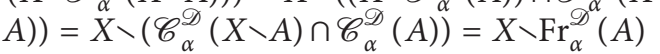

The converse of (1) and (4) of Theorem 7 is not true as shown in the following examples.

Example 2. Consider the topological space $(X, \tau)$ which is given in Example 1. Let $A=\{c\}$. Then $\operatorname{Fr}_{\alpha}(A)=\{c\} \nsubseteq \operatorname{Fr}_{\alpha}^{D}(A)=\phi$

Example 3. Let $(X, \tau)$ be a topological space, where $X=$ $\{a, b, c\}$ and $\tau=\{\phi,\{a, b\}, X\}$. Then $F_{X}=\{\phi,\{c\}$, $X, \mathscr{D} \alpha O(X)=\phi,\{a\},\{b\}, a, b,\{a, c,\{b, c, X, \mathscr{D} \alpha \mathscr{C}(X)=\phi$, $\{a\},\{b\},\{c\}, a, c, b, c, X$. Let $A=\{a, b\}$. Then $\operatorname{Fr}_{\alpha}^{D}(A)=$ $\{c\} \nsubseteq b r_{\alpha}^{\mathscr{D}}(A)=\phi$.

Theorem 8. Let $A$ be a subset of a topological space $X$. Then $\operatorname{Fr}_{\alpha}^{D}(A)=\phi$ if and only if $A$ is a $\mathscr{D} \alpha$-closed set and a $\mathscr{D} \alpha$-open set.

Proof. Suppose that $\operatorname{Fr}_{\alpha}^{\mathscr{D}}(A)=\phi$. First, we prove that $A$ is a $\mathscr{D} \alpha$-closed set. We have $\operatorname{Fr}_{\alpha}^{\mathscr{D}}(A)=\phi$ or $\mathscr{C}_{\alpha}^{\mathscr{D}}(A) \cap \mathscr{C}_{\alpha}^{\mathscr{D}}(X \backslash A)=\phi . \quad$ Hence, $\quad \mathscr{C}_{\alpha}^{\mathscr{D}}(A) \subseteq X \backslash \mathscr{C}_{\alpha}^{\mathscr{D}}$ $(X \backslash A)=\mathscr{I}_{\alpha}^{\mathscr{D}}(A)$. Therefore, $\mathscr{C}_{\alpha}^{\mathscr{D}}(A) \subseteq A$ and so $A$ is a $\mathscr{D} \alpha$-closed set. Now, we prove that $A$ is a $\mathscr{D} \alpha$-open set. Indeed, we have $\operatorname{Fr}_{\alpha}^{\mathscr{D}}(A)=\phi$ or $\mathscr{C}_{\alpha}^{\mathscr{D}}(A) \cap \mathscr{C}_{\alpha}^{\mathscr{D}}(X \backslash A)=\phi$. Hence, $A \cap\left(X \backslash \mathscr{I}_{\alpha}^{\mathscr{D}}(A)\right)=\phi$ and so $A \subseteq \mathscr{I}_{\alpha}^{\mathscr{D}}(A)$. Therefore, $A$ is a $\mathscr{D} \alpha$-open set. Conversely, suppose that $A$ is a $\mathscr{D} \alpha$-closed set and a $\mathscr{D} \alpha$-open set. Then $\operatorname{Fr}_{\alpha}^{\mathscr{D}}(A)=\mathscr{C}_{\alpha}^{\mathscr{D}}(A) \cap \mathscr{C}_{\alpha}^{\mathscr{D}}(X \backslash A)=\mathscr{C}_{\alpha}^{\mathscr{D}}(A) \cap\left(X \backslash \mathscr{I}_{\alpha}^{\mathscr{D}}(A)\right)=$ $A \cap(X \backslash A)=\phi$.

Theorem 9. Let $A$ be a subset of a topological space X. Then,

(1) $A$ is a $\mathscr{D} \alpha$-open set if and only if $A \cap \operatorname{Fr}_{\alpha}^{\mathscr{D}}(A)=\phi$;

(2) $A$ is a $\mathscr{D} \alpha$-closed set if and only if $\operatorname{Fr}_{\alpha}^{\mathscr{D}}(A) \subseteq A$.

Proof

(1) Let $A$ be a $\mathscr{D} \alpha$-open set. Then $\mathscr{I}_{\alpha}^{\mathscr{D}}(A)=A$ implies that $A \cap \operatorname{Fr}_{\alpha}^{\mathscr{D}}(A)=\mathscr{I}_{\alpha}^{\mathscr{D}}(A) \cap \operatorname{Fr}_{\alpha}^{\mathscr{D}}(A)=\phi$ (by Theorem 7 (3)). Conversely, suppose that $A \cap \operatorname{Fr}_{\alpha}^{\mathscr{D}}(A)=\phi$. Then $A \cap\left(\mathscr{C}_{\alpha}^{\mathscr{D}}(A) \cap \mathscr{C}_{\alpha}^{\mathscr{D}}(X \backslash A)\right)=\phi$ or $A \cap \mathscr{C}_{\alpha}^{\mathscr{D}}$ $(A) \cap \mathscr{C}_{\alpha}^{\mathscr{D}}(X \backslash A)=\phi \Rightarrow A \cap \mathscr{C}_{\alpha}^{\mathscr{D}}(X \backslash A)=\phi$, which implies that $A \subseteq X \backslash \mathscr{C}_{\alpha}^{\mathscr{D}}(X \backslash A)=\mathscr{I}_{\alpha}^{\mathscr{D}}(A)$. Moreover, $\mathscr{I}_{\alpha}^{\mathscr{D}}(A) \subseteq A$. Therefore, $\mathscr{I}_{\alpha}^{\mathscr{D}}(A)=A$ and thus $A$ is a $\mathscr{D} \alpha$-open set.

(2) Let $A$ be a $\mathscr{D} \alpha$-closed set. Then $\mathscr{C}_{\alpha}^{\mathscr{D}}(A)=A$. Now, $\operatorname{Fr}_{\alpha}^{D}(A)=\mathscr{C}_{\alpha}^{\mathscr{D}}(A) \cap \mathscr{C}_{\alpha}^{\mathscr{D}}(X \backslash A) \subseteq \mathscr{C}_{\alpha}^{\mathscr{D}}(A)=A$. That is, $\operatorname{Fr}_{\alpha}^{\mathscr{D}}(A) \subseteq A$. Conversely, suppose that $\operatorname{Fr}_{\alpha}^{D}(A) \subseteq A$. Then $\operatorname{Fr}_{\alpha}^{D}(A) \cap(X \backslash A)=\phi$. Since $\operatorname{Fr}_{\alpha}^{D}$ $(A)=\operatorname{Fr}_{\alpha}^{\mathscr{D}}(X \backslash A)$ (by Theorem 7 (8)), we have $\operatorname{Fr}_{\alpha}^{\mathscr{D}} X \backslash A \cap X \backslash A=\phi$. By (1), $X \backslash A$ is a $\mathscr{D} \alpha$-open set. Hence, $A$ is a $\mathscr{D} \alpha$-closed set.

Lemma 3. Let $A$ be a subset of a topological space $X$. If $A$ is a $\mathscr{D} \alpha$-closed set, then $A \backslash \mathscr{G}_{\alpha}^{\mathscr{D}}(A)=\operatorname{Fr}_{\alpha}^{D}(A)$.

Proof. It follows from ([15], Theorem 2.13) and Theorem 7 (14). 
Theorem 10. Let $A$ and $B$ be subsets of $X$. Then, the following results hold:

(1) $F r_{\alpha}^{\mathscr{D}}(A \cup B) \subseteq F r_{\alpha}^{\mathscr{D}}(A) \cup F r_{\alpha}^{\mathscr{D}}(B)$.

(2) $\operatorname{Fr}_{\alpha}^{\mathscr{D}}(A \cap B) \subseteq\left[\mathrm{Fr}_{\alpha}^{\mathscr{D}}(A) \cap \mathscr{C}_{\alpha}^{\mathscr{D}}(B)\right] \cup\left[\mathrm{Fr}_{\alpha}^{\mathscr{D}}(B) \cap\right.$ $\left.\mathscr{C}_{\alpha}^{D}(A)\right]$.
(3) $F r_{\alpha}^{\mathscr{D}}\left(F r_{\alpha}^{\mathscr{D}}\left(F r_{\alpha}^{\mathscr{D}}(A)\right)\right)=F r_{\alpha}^{\mathscr{D}}\left(F r_{\alpha}^{\mathscr{D}}(A)\right)$.

Proof.

$$
\text { (1) } \begin{aligned}
\mathrm{Fr}_{\alpha}^{\mathscr{D}}(A \cup B)= & \mathscr{C}_{\alpha}^{\mathscr{D}}(A \cup B) \cap \mathscr{C}_{\alpha}^{\mathscr{D}}(X \backslash A \cup B) \\
= & \left(\mathscr{C}_{\alpha}^{\mathscr{D}}(A) \cup \mathscr{C}_{\alpha}^{\mathscr{D}}(B)\right) \cap \mathscr{C}_{\alpha}^{\mathscr{D}}((X \backslash A) \cap(X \backslash B)) \\
\subseteq & \left(\mathscr{C}_{\alpha}^{\mathscr{D}}(A) \cup \mathscr{C}_{\alpha}^{\mathscr{D}}(B)\right) \cap\left[\mathscr{C}_{\alpha}^{\mathscr{D}}(X \backslash A) \cap \mathscr{C}_{\alpha}^{\mathscr{D}}(X \backslash B)\right] \\
= & \left(\mathscr{C}_{\alpha}^{\mathscr{D}}(A) \cap \mathscr{C}_{\alpha}^{\mathscr{D}}(X \backslash A)\right) \cap\left(\mathscr{C}_{\alpha}^{\mathscr{D}}(X \backslash B) \cup \mathscr{C}_{\alpha}^{\mathscr{D}}(B)\right) \cap\left[\mathscr{C}_{\alpha}^{\mathscr{D}}(X \backslash A) \cap \mathscr{C}_{\alpha}^{\mathscr{D}}(X \backslash B)\right] \\
= & \left(\mathrm{Fr}_{\alpha}^{\mathscr{D}}(A) \cap \mathscr{C}_{\alpha}^{\mathscr{D}}(X \backslash B)\right) \cup\left(\mathrm{Fr}_{\alpha}^{\mathscr{D}}(B) \cap \mathscr{C}_{\alpha}^{\mathscr{D}}(X \backslash A)\right) \subseteq \mathrm{Fr}_{\alpha}^{\mathscr{D}}(A) \cup \mathrm{Fr}_{\alpha}^{\mathscr{D}}(B) \\
& (2) \mathrm{Fr}_{\alpha}^{\mathscr{D}}(A \cap B)=\mathscr{C}_{\alpha}^{\mathscr{D}}(A \cap B) \cap \mathscr{C}_{\alpha}^{\mathscr{D}}(X \backslash A \cap B) \\
\subseteq & {\left[\mathscr{C}_{\alpha}^{\mathscr{D}}(A) \cap \mathscr{C}_{\alpha}^{\mathscr{D}}(B)\right] \cap\left[\mathscr{C}_{\alpha}^{\mathscr{D}}(X \backslash A) \cup(X \backslash B)\right] } \\
= & {\left[\mathscr{C}_{\alpha}^{\mathscr{D}}(A) \cap \mathscr{C}_{\alpha}^{\mathscr{D}}(B)\right] \cap\left[\mathscr{C}_{\alpha}^{\mathscr{D}}(X \backslash A) \cup \mathscr{C}_{\alpha}^{\mathscr{D}}(X \backslash B)\right] } \\
= & {\left[\mathscr{C}_{\alpha}^{\mathscr{D}}(A) \cap \mathscr{C}_{\alpha}^{\mathscr{D}}(B) \cap \mathscr{C}_{\alpha}^{\mathscr{D}}(X \backslash A)\right] \cup\left[\mathscr{C}_{\alpha}^{\mathscr{D}}(A) \cap \mathscr{C}_{\alpha}^{\mathscr{D}}(B) \cap \mathscr{C}_{\alpha}^{\mathscr{D}}(X \backslash B)\right] } \\
= & {\left[\operatorname{Fr}_{\alpha}^{\mathscr{D}}(A) \cap \mathscr{C}_{\alpha}^{\mathscr{D}}(B)\right] \cup\left[\mathscr{C}_{\alpha}^{\mathscr{D}}(A) \cap \mathrm{Fr}_{\alpha}^{\mathscr{D}}(B)\right] } \\
& (3) \operatorname{Fr}_{\alpha}^{\mathscr{D}}\left(\mathrm{Fr}_{\alpha}^{\mathscr{D}}\left(\mathrm{Fr}_{\alpha}^{\mathscr{D}}(A)\right)\right)=\mathscr{C}_{\alpha}^{\mathscr{D}}\left(\operatorname{Fr}_{\alpha}^{D}\left(\operatorname{Fr}_{\alpha}^{\mathscr{D}}(A)\right)\right) \cap \mathscr{C}_{\alpha}^{\mathscr{D}}\left(X \backslash \operatorname{Fr}_{\alpha}^{\mathscr{D}}\left(\operatorname{Fr}_{\alpha}^{\mathscr{D}}(A)\right)\right) \\
= & \operatorname{Fr}_{\alpha}^{\mathscr{D}}\left(\mathrm{Fr}_{\alpha}^{\mathscr{D}}(A)\right) \cap \mathscr{C}_{\alpha}^{\mathscr{D}}\left(X \backslash \mathrm{Fr}_{\alpha}^{\mathscr{D}}\left(\operatorname{Fr}_{\alpha}^{\mathscr{D}}(A)\right)\right)(\mathrm{i}) .
\end{aligned}
$$

Now consider

$$
\begin{aligned}
X \backslash\left(\mathrm{Fr}_{\alpha}^{\mathscr{D}}\left(\mathrm{Fr}_{\alpha}^{\mathscr{D}}(A)\right)\right) & =X \backslash\left[\mathscr{C}_{\alpha}^{\mathscr{D}}\left(\mathrm{Fr}_{\alpha}^{\mathscr{D}}(A)\right) \cap \mathscr{C}_{\alpha}^{\mathscr{D}}\left(X \backslash \mathrm{Fr}_{\alpha}^{\mathscr{D}}(A)\right)\right] \\
& =X \backslash\left[\mathrm{Fr}_{\alpha}^{\mathscr{D}}(A) \cap \mathscr{C}_{\alpha}^{\mathscr{D}}\left(X \backslash \mathrm{Fr}_{\alpha}^{\mathscr{D}}(A)\right)\right] \\
& =\left(X \backslash \mathrm{Fr}_{\alpha}^{\mathscr{D}}(A)\right) \cup\left(X \backslash \mathscr{C}_{\alpha}^{\mathscr{D}}\left(X \backslash \mathrm{Fr}_{\alpha}^{\mathscr{D}}(A)\right)\right) .
\end{aligned}
$$

Therefore,

$$
\begin{aligned}
\mathscr{C}_{\alpha}^{\mathscr{D}}\left(X \backslash \mathrm{Fr}_{\alpha}^{\mathscr{D}}\left(\mathrm{Fr}_{\alpha}^{\mathscr{D}}(A)\right)\right) & =\mathscr{C}_{\alpha}^{\mathscr{D}}\left[\mathscr{C}_{\alpha}^{\mathscr{D}}\left(X \backslash \mathrm{Fr}_{\alpha}^{\mathscr{D}}(A)\right) \cup X \backslash \mathscr{C}_{\alpha}^{\mathscr{D}} X \backslash \mathrm{Fr}_{\alpha}^{\mathscr{D}}(A)\right] \\
& =\mathscr{C}_{\alpha}^{\mathscr{D}}\left(\mathscr{C}_{\alpha}^{\mathscr{D}}\left(X \backslash \mathrm{Fr}_{\alpha}^{\mathscr{D}}(A)\right)\right) \cup \mathscr{C}_{\alpha}^{\mathscr{D}}\left(X \backslash \mathscr{C}_{\alpha}^{\mathscr{D}}\left(X \backslash \mathrm{Fr}_{\alpha}^{\mathscr{D}}(A)\right)\right) \\
& =B \cup\left(X \backslash \mathscr{C}_{\alpha}^{\mathscr{D}}\left(X \backslash \mathrm{Fr}_{\alpha}^{\mathscr{D}}(B)\right)\right)=X(\mathrm{ii}),
\end{aligned}
$$

where $B=\mathscr{C}_{\alpha}^{\mathscr{D}} \mathscr{C}_{\alpha}^{\mathscr{D}} X \backslash \mathrm{Fr}_{\alpha}^{\mathscr{D}}(A)$. From (i) and (ii), we have

$$
\operatorname{Fr}_{\alpha}^{\mathscr{D}}\left(\operatorname{Fr}_{\alpha}^{\mathscr{D}}\left(\operatorname{Fr}_{\alpha}^{\mathscr{D}}(A)\right)\right)=\operatorname{Fr}_{\alpha}^{\mathscr{D}}\left(\operatorname{Fr}_{\alpha}^{\mathscr{D}}(A)\right) \cap X=\operatorname{Fr}_{\alpha}^{\mathscr{D}}\left(\operatorname{Fr}_{\alpha}^{\mathscr{D}}(A)\right) \text {. }
$$

Definition 8. Let $A$ be a subset of a topological space $X$. The $\mathscr{D} \alpha$-exterior of $A$, denoted by $\operatorname{Ext}_{\alpha}^{\mathscr{D}}(A)$, is defined as $\operatorname{Ext}_{\alpha}^{\mathscr{D}}(A)=\mathscr{I}_{\alpha}^{\mathscr{D}}(X \backslash A)$.

Theorem 11. Let $A$ and $B$ be subsets of $X$. Then the following results hold:
(1) $\operatorname{Ext}_{\alpha}(A) \subseteq \operatorname{Ext}_{\alpha}^{D}(A)$, where $\operatorname{Ext}_{\alpha}(A)$ is the $\alpha$-exterior ([22], Definition 2.16) of $A$.

(2) $\operatorname{Ext}_{\alpha}^{\mathscr{D}}(A)=X \backslash \mathscr{C}_{\alpha}^{\mathscr{D}}(A)$.

(3) $\operatorname{Ext}_{\alpha}^{\mathscr{D}}\left(\operatorname{Ext}_{\alpha}^{\mathscr{D}}(A)\right)=\mathscr{I}_{\alpha}^{\mathscr{D}}\left(\mathscr{C}_{\alpha}^{\mathscr{D}}(A)\right)$.

(4) If $A \subseteq B$, then $\operatorname{Ext}_{\alpha}^{\mathscr{D}}(B) \subseteq \operatorname{Ext}_{\alpha}^{\mathscr{D}}(A)$.

(5) $\operatorname{Ext}_{\alpha}^{\mathscr{D}}(A \cup B) \subseteq \operatorname{Ext}_{\alpha}^{\mathscr{D}}(A) \cap \operatorname{Ext}_{\alpha}^{\mathscr{D}}(B)$.

(6) $\operatorname{Ext}_{\alpha}^{\mathscr{D}}(A \cap B) \supseteq \operatorname{Ext}_{\alpha}^{\mathscr{D}}(A) \cup E x t_{\alpha}^{\mathscr{D}}(B)$.

(7) $\operatorname{Ext}_{\alpha}^{\mathscr{D}}(X)=\phi$ and $\operatorname{Ext}_{\alpha}^{\mathscr{D}}(\phi)=X$.

(8) $\operatorname{Ext}_{\alpha}^{\mathscr{D}}(A)=\operatorname{Ext}_{\alpha}^{\mathscr{D}}\left(X \backslash \operatorname{Ext}_{\alpha}^{\mathscr{D}}(A)\right)$.

(9) $X=\mathscr{F}_{\alpha}^{\mathscr{D}}(A) \cup \operatorname{Ext}_{\alpha}^{\mathscr{D}}(A) \cup F r_{\alpha}^{\mathscr{D}}(A)$. 
Proof.

(1) It follows from ([15], Theorem 3.15 (i)).

(2) It follows from ([15], Lemma 3.13 (i)).

(3) It follows from ([15], Lemma 3.13 (ii)).

(4) It follows from ([15], Theorem 3.15 (iii)).

(5) It follows from ([15], Theorem 3.15 (vi)).

(6) It follows from ([15], Theorem 3.15 (v)).

(7) It is obvious.

(8) It follows from ([15], Theorem 3.15 (iv)).

(9) It is obvious.

The opposite of (1) and (4) of Theorem 11 is not true as shown in the following examples.

Example 4. Consider the topological space $(X, \tau)$ which is given in Example 1. Let $A=\{a\}$. Then $\operatorname{Ext}_{\alpha}^{\mathscr{D}}(A)=\{b, c\} \nsubseteq \operatorname{Ext}_{\alpha}(A)=\phi$.

Example 5. Consider the topological space $(X, \tau)$ which is given in Example 3. Let $A=\{a\}$ and $B=\{a, b\}$. Then $\operatorname{Ext}_{\alpha}^{D}(A)=b, c \nsubseteq \operatorname{Ext}_{\alpha}^{\mathscr{D}}(B)=\phi$.

Remark 1. The equality in statements (5) of Theorem 11 need not be true as seen from Example 3 . Let $A=\{a\}, B=\{b\}$, and $A \cup B=\{a, b\}$. Then $\operatorname{Ext}_{\alpha}^{D}(A \cup B)=$ $\phi \neq\{c\}=\operatorname{Ext}_{\alpha}^{\mathscr{D}}(A) \cap \operatorname{Ext}_{\alpha}^{\mathscr{D}}(B)$. Furthermore, the equality in statement (6) of the above theorem need not be true as seen from Example 3. Let $A=\{a, b\}, B=\{c\}$, and $A \cap B=\phi$. Then $\operatorname{Ext}_{\alpha}^{\mathscr{D}}(A \cap B)=X \neq\{a, b\}=\operatorname{Ext}_{\alpha}^{\mathscr{D}}(A) \cup \operatorname{Ext}_{\alpha}^{\mathscr{D}}(B)$.

\section{3. $D \boldsymbol{\alpha}-R_{0}$ and $\mathscr{D} \boldsymbol{\alpha}-R_{1}$ Spaces}

Definition 9. Let $A$ be a subset of a topological space $X$. The $\mathscr{D} \alpha$-kernel of $A$, denoted by $\operatorname{Ker}_{\alpha}^{\mathscr{D}}(A)$, is defined as $\operatorname{Ker}_{\alpha}^{D}(A)=\cap\{U \in \mathscr{D} \alpha O(X) \mid A \subset U\}$.

Definition 10. Let $x$ be a point of a topological space $X$. The $\mathscr{D} \alpha$-kernel of $x$, denoted by $\operatorname{Ker}_{\alpha}^{\mathscr{D}}(\{x\})$, is defined as $\operatorname{Ker}_{\alpha}^{\mathscr{D}}(\{x\})=\cap\{U \in \mathscr{D} \alpha O(X) \mid x \in U\}$.

Lemma 4. Let $(X, \tau)$ be a topological space and $x \in X$.Then,

(1) $y \in \operatorname{Ker}_{\alpha}^{\mathscr{D}}(\{x\})$ if and only if $x \in \mathscr{C}_{\alpha}^{\mathscr{D}}(\{y\})$;

(2) $\operatorname{Ker}_{\alpha}^{\mathscr{D}}(A)=\cap\left\{x \in X \mid \mathscr{C}_{\alpha}^{\mathscr{D}}(\{x\}) \cap A \neq \phi\right\}$.

Proof

(1) Suppose that $y \notin \operatorname{Ker}_{\alpha}^{\mathscr{D}}(\{x\})$. Then there exists a $\mathscr{D} \alpha$-open set $V$ containing $x$ such that $y \notin V$. Therefore, we have $x \notin \mathscr{C}_{\alpha}^{\mathscr{D}}(\{y\})$. The proof of the opposite case can be done similarly.

(2) Let $x \in \operatorname{Ker}_{\alpha}^{\mathscr{D}}(A)$ and $\mathscr{C}_{\alpha}^{\mathscr{D}}(\{x\}) \cap A=\phi$. Hence, $x \notin X-\mathscr{C}_{\alpha}^{\mathscr{D}}(\{x\})$ which is a $\mathscr{D} \alpha$-open set containing $A$. This is impossible, since $x \in \operatorname{Ker}_{\alpha}^{\mathscr{D}}(A)$. Consequently, $\mathscr{C}_{\alpha}^{\mathscr{D}}(\{x\}) \cap A \neq \phi$. Let $\mathscr{C}_{\alpha}^{\mathscr{D}}(\{x\}) \cap A \neq \phi$ and $x \notin \operatorname{Ker}_{\alpha}^{\mathscr{D}}(A)$. Then, there exists a $\mathscr{D} \alpha$-open set $W$ containing $A$ and $x \notin W$. Let $y \notin \mathscr{C}_{\alpha}^{\mathscr{D}}(\{x\}) \cap A$.
Hence, $W$ is a $\mathscr{D} \alpha$-neighborhood of $y$ where $x \notin W$. By this contradiction, $x \in \operatorname{Ker}_{\alpha}^{\mathscr{D}}(A)$ and the proof is completed.

Lemma 5. The following statements are equivalent for any points $x$ and $y$ in a topological space $(X, \tau)$ :

(1) $\operatorname{Ker}_{\alpha}^{\mathscr{D}}(\{x\}) \neq \operatorname{Ker}_{\alpha}^{\mathscr{D}}(\{y\})$.

(2) $\mathscr{C}_{\alpha}^{\mathscr{D}}(\{x\}) \neq \mathscr{C}_{\alpha}^{\mathscr{D}}(\{y\})$.

Proof

(i) $(1) \Rightarrow(2)$ Suppose that $\operatorname{Ker}_{\alpha}^{\mathscr{D}}(\{x\}) \neq \operatorname{Ker}_{\alpha}^{\mathscr{D}}(\{y\})$. Then there exists a point $z$ in $X$ such that $z \in \operatorname{Ker}_{\alpha}^{D}(\{x\})$ and $z \notin \operatorname{Ker}_{\alpha}^{D}(\{y\})$. It follows from $z \in \operatorname{Ker}_{\alpha}^{\mathscr{D}}(\{x\})$ that $\{x\} \cap \mathscr{C}_{\alpha}^{\mathscr{D}}(\{z\}) \neq \phi$. This implies that $x \in \mathscr{C}_{\alpha}^{\mathscr{D}}(\{z\})$. By $z \notin \operatorname{Ker}_{\alpha}^{\mathscr{D}}(\{y\})$, we have $\{y\} \cap \mathscr{C}_{\alpha}^{\mathscr{D}}(\{z\})=\phi$. Since $x \in \mathscr{C}_{\alpha}^{\mathscr{D}}(\{z\}), \mathscr{C}_{\alpha}^{\mathscr{D}}(\{x\}) \subset$ $\mathscr{C}_{\alpha}^{\mathscr{D}}(\{z\}) \quad$ and $\quad\{y\} \cap \mathscr{C}_{\alpha}^{\mathscr{D}}(\{x\})=\phi, \quad \mathscr{C}_{\alpha}^{\mathscr{D}}(\{x\}) \neq$ $\mathscr{C}_{\alpha}^{\mathscr{D}}(\{y\})$. Now, $\operatorname{Ker}_{\alpha}^{\mathscr{D}}(\{x\}) \neq \operatorname{Ker}_{\alpha}^{\mathscr{D}}(\{y\})$ implies that $\mathscr{C}_{\alpha}^{\mathscr{D}}(\{x\}) \neq \mathscr{C}_{\alpha}^{\mathscr{D}}(\{y\})$.

(ii) $(2) \Rightarrow$ (1) Suppose that $\mathscr{C}_{\alpha}^{\mathscr{D}}(\{x\}) \neq \mathscr{C}_{\alpha}^{\mathscr{D}}(\{y\})$. Then there exists a point $z$ in $X$ such that $z \in \mathscr{C}_{\alpha}^{\mathscr{D}}(\{x\})$ and $z \notin \mathscr{C}_{\alpha}^{\mathscr{D}}(\{y\})$. Then, there exists a $\mathscr{D} \alpha$-open set containing $z$ and therefore $x$ but not $y$, that is, $y \notin \operatorname{Ker}_{\alpha}^{\mathscr{D}}(\{x\})$. Hence, $\operatorname{Ker}_{\alpha}^{\mathscr{D}}(\{x\}) \neq \operatorname{Ker}_{\alpha}^{\mathscr{D}}(\{y\})$.

Definition 11. A topological space $(X, \tau)$ is said to be a $\mathscr{D} \alpha-$ $R_{0}$ space if every $\mathscr{D} \alpha$-open set contains the $\mathscr{D} \alpha$-closure of each of its singletons.

Theorem 12. Let $(X, \tau)$ be a topological space. Then,

(1) every $\alpha-R_{0}$ space is $\mathscr{D} \alpha-R_{0}$

(2) every $g-R_{0}$ space is $\mathscr{D} \alpha-R_{0}$

Proof. It is obvious from ([15], Theorem 3.6).

From the above discussions, we have the following diagram in which the opposites of implications need not be true.

$$
\alpha-R_{0} \longrightarrow \mathscr{D} \alpha-R_{0} \longleftarrow g-R_{0}
$$

Theorem 13. A topological space $(X, \tau)$ is a $\mathscr{D} \alpha-R_{0}$ space if and only if, for any $x$ and $y$ in $X, \mathscr{C}_{\alpha}^{\mathscr{D}}(\{x\}) \neq \mathscr{C}_{\alpha}^{\mathscr{D}}(\{y\})$ implies that $\mathscr{C}_{\alpha}^{\mathscr{D}}(\{x\}) \cap \mathscr{C}_{\alpha}^{\mathscr{D}}(\{y\})=\phi$.

Proof.

Necessity. Suppose that $(X, \tau)$ is a $\mathscr{D} \alpha-R_{0}$ and $x, y \in X$ such that $\mathscr{C}_{\alpha}^{\mathscr{D}}(\{x\}) \neq \mathscr{C}_{\alpha}^{\mathscr{D}}(\{y\})$. Then, there exists $z \in \mathscr{C}_{\alpha}^{\mathscr{D}}(\{x\})$ such that $z \notin \mathscr{C}_{\alpha}^{\mathscr{D}}(\{y\})$ (or $z \in \mathscr{C}_{\alpha}^{\mathscr{D}}(\{y\})$ such that $\left.z \notin \mathscr{C}_{\alpha}^{\mathscr{D}}(\{x\})\right)$. There exists $U \in \mathscr{D} \alpha O(X)$ such that $y \notin U$ and $z \in U$; hence, $x \in U$. Therefore, we have $x \notin \mathscr{C}_{\alpha}^{\mathscr{D}}(\{y\})$. Thus, $x \in X-\mathscr{C}_{\alpha}^{\mathscr{D}}(\{y\}) \in \mathscr{D} \alpha O(X)$, which implies that 
$\mathscr{C}_{\alpha}^{\mathscr{D}}(\{x\}) \subset X-\mathscr{C}_{\alpha}^{\mathscr{D}}(\{y\})$ and $\mathscr{C}_{\alpha}^{\mathscr{D}}(\{x\}) \cap \mathscr{C}_{\alpha}^{\mathscr{D}}(\{y\})=$ $\phi$. The proof for otherwise is similar.

(i) Sufficiency. Let $U \in \mathscr{D} \alpha O(X)$ and $x \in U$. We will show that $\mathscr{C}_{\alpha}^{\mathscr{D}}(\{x\}) \subset U$. Let $y \notin U$; that is, $y \in X-U$. Then $x \neq y$ and $x \notin \mathscr{C}_{\alpha}^{D}(\{y\})$. This shows that $\mathscr{C}_{\alpha}^{\mathscr{D}}(\{x\}) \neq \mathscr{C}_{\alpha}^{\mathscr{D}}(\{y\})$. By assumption, $\mathscr{C}_{\alpha}^{\mathscr{D}}(\{x\}) \cap \mathscr{C}_{\alpha}^{\mathscr{D}}(\{y\})=\phi$. Hence, $y \notin \mathscr{C}_{\alpha}^{\mathscr{D}}(\{x\})$ and therefore $\mathscr{C}_{\alpha}^{D^{\alpha}}(\{x\}) \subset U$.

Theorem 14. A topological space $(X, \tau)$ is a $\mathscr{D} \alpha-R_{0}$ space if and only if, for any $x$ and $y$ in $X, \operatorname{Ker}_{\alpha}^{D}(\{x\}) \neq \operatorname{Ker}_{\alpha}^{\mathscr{D}}(\{y\})$ implies that $\operatorname{Ker}_{\alpha}^{\mathscr{D}}(\{x\}) \cap \operatorname{Ker}_{\alpha}^{D}(\{x\})=\phi$.

Proof. Suppose that $(X, \tau)$ is a $\mathscr{D} \alpha-R_{0}$ space. Then, by Lemma 5, for any points $x$ and $y$ in $X$ if $\operatorname{Ker}_{\alpha}^{\mathscr{D}}(\{x\}) \neq \operatorname{Ker}_{\alpha}^{\mathscr{D}}(\{y\})$, then $\mathscr{C}_{\alpha}^{\mathscr{D}}(\{x\}) \neq \mathscr{C}_{\alpha}^{\mathscr{D}}(\{y\})$. Now, we prove that $\operatorname{Ker}_{\alpha}^{\mathscr{D}}(\{x\}) \cap \operatorname{Ker}_{\alpha}^{\mathscr{D}}(\{y\})=\phi$. Assume that $z \in \operatorname{Ker}_{\alpha}^{\mathscr{D}}(\{x\}) \cap \operatorname{Ker}_{\alpha}^{\mathscr{D}}(\{y\})$. By $z \in \operatorname{Ker}_{\alpha}^{\mathscr{D}}(\{x\})$ and Lemma 4 (1), it follows that $x \in \mathscr{C}_{\alpha}^{\mathscr{D}}(\{z\})$. Since $x \in \mathscr{C}_{\alpha}^{\mathscr{D}}(\{x\})$, by Theorem $13 \mathscr{C}_{\alpha}^{\mathscr{D}}(\{x\})=\mathscr{C}_{\alpha}^{\mathscr{D}}(\{z\})$. Similarly, we have $\mathscr{C}_{\alpha}^{\mathscr{D}}(\{y\})=\mathscr{C}_{\alpha}^{\mathscr{D}}(\{z\})=\mathscr{C}_{\alpha}^{\mathscr{D}}(\{x\})$. This is a contradiction. Therefore, we have $\operatorname{Ker}_{\alpha}^{\mathscr{D}}(\{x\}) \cap \operatorname{Ker}_{\alpha}^{\mathscr{D}}(\{y\})=\phi$. Conversely, let $(X, \tau)$ be a topological space such that, for any points $x$ and $y$ in $X, \operatorname{Ker}_{\alpha}^{D}(\{x\}) \neq \operatorname{Ker}_{\alpha}^{D}(\{y\})$ implies that $\operatorname{Ker}_{\alpha}^{\mathscr{D}}(\{x\}) \cap \operatorname{Ker}_{\alpha}^{\mathscr{D}}(\{y\})=\phi$. If $\mathscr{C}_{\alpha}^{\mathscr{D}}(\{x\}) \neq \mathscr{C}_{\alpha}^{\mathscr{D}}(\{y\})$, then, by Lemma $5, \quad \operatorname{Ker}_{\alpha}^{\mathscr{D}}(\{x\}) \neq \operatorname{Ker}_{\alpha}^{\mathscr{D}}(\{x\})$. Hence, $\operatorname{Ker}_{\alpha}^{D}(\{x\}) \cap \operatorname{Ker}_{\alpha}^{\mathscr{D}}(\{y\})=\phi$, which implies that $\mathscr{C}_{\alpha}^{\mathscr{D}}(\{x\}) \cap \mathscr{C}_{\alpha}^{\mathscr{D}}(\{y\})=\phi$. Because $z \in \mathscr{C}_{\alpha}^{\mathscr{D}}(\{x\})$ implies that $x \in \operatorname{Ker}_{\alpha}^{\mathscr{D}}(\{z\}), \operatorname{Ker}_{\alpha}^{\mathscr{D}}(\{x\}) \cap \operatorname{Ker}_{\alpha}^{\mathscr{D}}(\{z\}) \neq \phi$. By hypothesis, we have $\operatorname{Ker}_{\alpha}^{\mathscr{D}}(\{x\})=\operatorname{Ker}_{\alpha}^{\mathscr{D}}(\{z\})$. Then $z \in \mathscr{C}_{\alpha}^{\mathscr{D}}(\{x\}) \cap$ $\mathscr{C}_{\alpha}^{\mathscr{D}}(\{x\})$ implies that $\operatorname{Ker}_{\alpha}^{\mathscr{D}}(\{x\})=\operatorname{Ker}_{\alpha}^{\mathscr{D}}(\{z\})=\operatorname{Ker}_{\alpha}^{\mathscr{D}}(\{y\})$. This is a contradiction. Hence, $\mathscr{C}_{\alpha}^{\mathscr{D}}(\{x\}) \cap \mathscr{C}_{\alpha}^{\mathscr{D}}(\{y\})=\phi$. By Theorem 13, we have that $(X, \tau)$ is a $\mathscr{D} \alpha-R_{0}$ space.

Theorem 15. For a topological space $(X, \tau)$, the following properties are equivalent:

(1) $(X, \tau)$ is a $\mathscr{D} \alpha-R_{0}$ space.

(2) For any $A \neq \phi$ and $G \in \mathscr{D} \alpha O(X)$ such that $A \cap G \neq \phi$, there exists $F \in \mathscr{D} \alpha \mathscr{C}(X)$ such that $A \cap F \neq \phi$ and $F \subset G$.

(3) Any $A \in \mathscr{D} \alpha O(X), G=\cup\{F \in \mathscr{D} \alpha \mathscr{C}(X) \mid F \subset G\}$.

(4) Any $F \in \mathscr{D} \alpha O(X), F=\cap\{G \in \mathscr{D} \alpha O(X) \mid F \subset G\}$.

(5) For any $x \in X, \mathscr{C}_{\alpha}^{\mathscr{D}}(\{x\}) \subset \operatorname{Ker}_{\alpha}^{\mathscr{D}}(\{x\})$.

Proof. $\quad(1) \Rightarrow(2)$ Let $A$ be a nonempty set of $X$ and $G \in \mathscr{D} \alpha O(X)$ such that $A \cap G \neq \phi$. There exists $x \in A \cap G$. Since $\quad x \in G \in \quad \mathscr{D} \alpha O(X)$, $\mathscr{C}_{\alpha}^{\mathscr{D}}(\{x\}) \subset G$. Set $\quad F=\mathscr{C}_{\alpha}^{\mathscr{D}}(\{x\}) ; \quad$ then $F \in \mathscr{D} \alpha \mathscr{C}(X), F \subset G$, and $A \cap F \neq \phi$.

$(2) \Rightarrow(3)$ Let $G \in \mathscr{D} \alpha O(X)$. Then $G^{\cup\{F \in \mathscr{D} \alpha \mathscr{C}(X) \mid F \subset G\}}$. Let $x$ be any point of $G$. There exists $F \in \mathscr{D} \alpha \mathscr{C}(X)$ such that $x \in F$ and $F \subset G$. Therefore, we have $x \in F \subset \cup\{F \in \mathscr{D} \alpha \mathscr{C}(X) \mid F \subset G\}$ and hence $G=\cup\{F \in \mathscr{D} \alpha \mathscr{C}(X) \mid F \subset G\}$.

$(3) \Rightarrow(4)$ This is obvious.
$(4) \Rightarrow(5)$ Let $x$ be any point of $X$ and $y \notin \operatorname{Ker}_{\alpha}^{\mathscr{D}}(\{x\})$. There exists $U \in \mathscr{D} \alpha O(X)$ such that $x \in U$ and $y \notin U$. Hence, $\mathscr{C}_{\alpha}^{\mathscr{D}}(\{y\}) \cap U=\phi$. By (4) $\cap G \in \mathscr{D} \alpha O(X) \mid$ $\mathscr{C}_{\alpha}^{D}(\{y\}) \subset G \cap U=\phi$. There exists $G \in \mathscr{D} \alpha O(X)$ such that $x \notin G$ and $\mathscr{C}_{\alpha}^{\mathscr{D}}(\{y\}) \subset G$. Therefore, $\mathscr{C}_{\alpha}^{\mathscr{D}}(\{x\}) \cap G=\phi$ and $y \notin \mathscr{C}_{\alpha}^{\mathscr{D}}(\{x\})$. Consequently, we obtain $\mathscr{C}_{\alpha}^{\mathscr{D}}(\{x\}) \subset \operatorname{Ker}_{\alpha}^{\mathscr{D}}(\{x\})$.

$(5) \Rightarrow(1)$ Let $G \in \mathscr{D} \alpha O(X)$ and $x \in G$. Suppose that $y \in \operatorname{Ker}_{\alpha}^{\mathscr{D}}(\{x\})$. Then $x \in \mathscr{C}_{\alpha}^{\mathscr{D}}(\{y\})$ and $y \in G$. This implies that $\mathscr{C}_{\alpha}^{\mathscr{D}}(\{x\}) \subset \operatorname{Ker}_{\alpha}^{\mathscr{D}}(\{x\}) \subset G$. Therefore, $(X, \tau)$ is a $\mathscr{D} \alpha-R_{0}$ space.

Corollary 2. For a topological space $(X, \tau)$, the following properties are equivalent:

(1) $(X, \tau)$ is a $\mathscr{D} \alpha-R_{0}$ space.

(2) $\mathscr{C}_{\alpha}^{\mathscr{D}}(\{x\})=\operatorname{Ker}_{\alpha}^{\mathscr{D}}(\{x\})$ for all $x \in X$.

Proof

$(1) \Rightarrow(2)$ Suppose that $(X, \tau)$ is a $\mathscr{D} \alpha-R_{0}$ space. By Theorem 15, $\mathscr{C}_{\alpha}^{\mathscr{D}}(\{x\}) \subset \operatorname{Ker}_{\alpha}^{\mathscr{D}}(\{x\})$ for each $x \in X$. Let $y \in \operatorname{Ker}_{\alpha}^{D}(\{x\})$. Then $x \in \mathscr{C}_{\alpha}^{\mathscr{D}}(\{x\})$ and so $\mathscr{C}_{\alpha}^{\mathscr{D}}(\{x\})=\mathscr{C}_{\alpha}^{\mathscr{D}}(\{y\})$. Therefore, $y \in \mathscr{C}_{\alpha}^{\mathscr{D}}(\{x\})$ and hence $\operatorname{Ker}_{\alpha}^{\mathscr{D}}(\{x\}) \subset \mathscr{C}_{\alpha}^{\mathscr{D}}(\{x\})$. This shows that $\mathscr{C}_{\alpha}^{\mathscr{D}}(\{x\})=\operatorname{Ker}_{\alpha}^{\mathscr{D}}(\{x\})$.

$(2) \Rightarrow(1)$ This is obvious by Theorem 15 .

Theorem 16. For a topological space $(X, \tau)$, the following properties are equivalent:

(1) $(X, \tau)$ is a $\mathscr{D} \alpha-R_{0}$ space.

(2) $x \in \mathscr{C}_{\alpha}^{\mathscr{D}}(\{y\})$ if and only if $y \in \mathscr{C}_{\alpha}^{\mathscr{D}}(\{x\})$, for any points $x$ and $y$ in $X$.

Proof

$(1) \Rightarrow(2)$ Assume that $(X, \tau)$ is a $\mathscr{D} \alpha-R_{0}$ space. Let $x \in \mathscr{C}_{\alpha}^{\mathscr{D}}(\{y\})$ and let $W$ be any $\mathscr{D} \alpha$-open set such that $y \in W$. Now, by hypothesis, $x \in W$. Therefore, every $\mathscr{D} \alpha$-open set containing $y$ contains $x$. Hence, $y \in \mathscr{C}_{\alpha}^{\mathscr{D}}(\{x\})$.

(2) $\Rightarrow(1)$ Let $U$ be a $\mathscr{D} \alpha$-open set and $x \in U$. If $y \notin U$, then $x \notin \mathscr{C}_{\alpha}^{\mathscr{D}}(\{y\})$ and hence $y \notin \mathscr{C}_{\alpha}^{\mathscr{D}}(\{x\})$. This implies that $\mathscr{C}_{\alpha}^{\mathscr{D}}(\{x\}) \subset U$. Hence, $(X, \tau)$ is a $\mathscr{D} \alpha-R_{0}$ space.

Theorem 17. For a topological space $(X, \tau)$, the following properties are equivalent:

(1) $(X, \tau)$ is a $\mathscr{D} \alpha-R_{0}$ space.

(2) If $F$ is $\mathscr{D} \alpha$-closed, then $F=\operatorname{Ker}_{\alpha}^{D}(F)$.

(3) If $F$ is $\mathscr{D} \alpha$-closed and $x \in F$, then $\operatorname{Ker}_{\alpha}^{\mathscr{D}}(\{x\}) \subset F$.

(4) If $x \in X$, then $\operatorname{Ker}_{\alpha}^{\mathscr{D}}(\{x\}) \subset \mathscr{C}_{\alpha}^{\mathscr{D}}(\{x\})$. 
$(1) \Rightarrow(2)$ Let $F$ be $\mathscr{D} \alpha$-closed and $x \notin F$. Thus, $X-F$ is $\mathscr{D} \alpha$-open and contains $x$. Since $(X, \tau)$ is $\mathscr{D} \alpha-R_{0}, \mathscr{C}_{\alpha}^{\mathscr{D}}(\{x\}) \subset X-F$. Thus $\mathscr{C}_{\alpha}^{\mathscr{D}}(\{x\}) \cap F=\phi$ and by Lemma 4 (2) $x \notin \operatorname{Ker}_{\alpha}^{\mathscr{D}}(F)$. Therefore, $\operatorname{Ker}_{\alpha}^{\mathscr{D}}(F)=F$. $(2) \Rightarrow(3) \quad$ In general, $A \subset B$ implies that $\operatorname{Ker}_{\alpha}^{\mathscr{D}}(A) \subset \operatorname{Ker}_{\alpha}^{\mathscr{D}}(B)$. Therefore, it follows from (2) that $\operatorname{Ker}_{\alpha}^{\mathscr{D}}(\{x\}) \subset \operatorname{Ker}_{\alpha}^{\mathscr{D}}(F)=F$.

$(3) \Rightarrow(4) \quad$ Since $\quad x \in \mathscr{C}_{\alpha}^{\mathscr{D}}(\{x\})$ and $\mathscr{C}_{\alpha}^{\mathscr{D}}(\{x\})$ is $\mathscr{D} \alpha$-closed, by (3), $\operatorname{Ker}_{\alpha}^{\mathscr{D}}(\{x\}) \subset \mathscr{C}_{\alpha}^{\mathscr{D}}(\{x\})$.

$(4) \Rightarrow$ (1) We show the implication by using Theorem 16. Let $x \in \mathscr{C}_{\alpha}^{\mathscr{D}}(\{x\})$. Then, by Lemma 4 (1), $y \in \operatorname{Ker}_{\alpha}^{\mathscr{D}}(\{x\})$. Since $x \in \mathscr{C}_{\alpha}^{\mathscr{D}}(\{x\})$ and $\mathscr{C}_{\alpha}^{\mathscr{D}}(\{x\})$ is $\mathscr{D} \alpha$-closed, by (4), we obtain $y \in \operatorname{Ker}_{\alpha}^{\mathscr{D}}(\{x\}) \subset \mathscr{C}_{\alpha}^{\mathscr{D}}(\{x\})$. Therefore, $x \in \mathscr{C}_{\alpha}^{\mathscr{D}}(\{y\})$ implies that $y \in \mathscr{C}_{\alpha}^{\mathscr{D}}(\{x\})$. The opposite is obvious and $(X, \tau)$ is a $\mathscr{D} \alpha-R_{0}$ space.

Definition 12. A filter base $F$ is called $\mathscr{D} \alpha$-convergent to a point $x$ in $X$, if, for any $\mathscr{D} \alpha$-open set $U$ of $X$ containing $x$, there exists $B$ in $F$ such that $B$ is a subset of $U$.

Lemma 6. Let $(X, \tau)$ be a topological space and $x$ and $y$ are any two points in $X$ such that every net in $X \mathscr{D} \alpha$-converging to $y \mathscr{D} \alpha$-converges to $x$. Then $x \in \mathscr{C}_{\alpha}^{\mathscr{D}}(\{y\})$.

Proof. Suppose that $x_{n}=y$ for each $n \in \mathbf{N}$. Then $\left\{x_{n}\right\}_{n \in \mathbf{N}}$ is a net in $\mathscr{C}_{\alpha}^{\mathscr{D}}(\{y\})$. Since $\left\{x_{n}\right\}_{n \in \mathbf{N}} \mathscr{D} \alpha$-converges to $y$, $\left\{x_{n}\right\}_{n \in \mathrm{N}} \mathscr{D} \alpha$-converges to $x$ and this implies that $x \in \mathscr{C}_{\alpha}^{D}(\{y\})$.

Theorem 18. For a topological space $(X, \tau)$, the following statements are equivalent:

(1) $(X, \tau)$ is a $\mathscr{D} \alpha-R_{0}$ space.

(2) If $x, y \in X$, then $y \in \mathscr{C}_{\alpha}^{\mathscr{D}}(\{x\})$ if and only if every net in $X \mathscr{D} \alpha$-converging to $y \mathscr{D} \alpha$-converges to $x$.

\section{Proof}

$(1) \Rightarrow(2)$ Let $x, y \in X$ such that $y \in \mathscr{C}_{\alpha}^{\mathscr{D}}(\{x\})$. Suppose that $\left\{x_{\alpha}\right\}_{\alpha \in \mathbf{N}}$ is a net in $X$ such that $\left\{x_{\alpha}\right\}_{\alpha \in \mathbf{N}} \mathscr{D} \alpha$-converges to $y$. Since $y \in \mathscr{C}_{\alpha}^{\mathscr{D}}(\{x\})$ and by Theorem 15 , we have $\mathscr{C}_{\alpha}^{\mathscr{D}}(\{x\})=\mathscr{C}_{\alpha}^{\mathscr{D}}(\{y\})$. Therefore, $x \in \mathscr{C}_{\alpha}^{\mathscr{D}}(\{y\})$. This means that $\left\{x_{\alpha}\right\}_{\alpha \in \Lambda} \mathscr{D} \alpha$-converges to $x$. Conversely, let $x, y \in X$ such that every net in $X \mathscr{D} \alpha$-converging to $y \mathscr{D} \alpha$-converges to $x$. Then $x \in \mathscr{C}_{\alpha}^{\mathscr{D}}(\{y\})$ by Lemma 4 (2). By Theorem 15, we have $\mathscr{C}_{\alpha}^{\mathscr{D}}(\{x\})=\mathscr{C}_{\alpha}^{\mathscr{D}}(\{y\})$. Therefore, $y \in \mathscr{C}_{\alpha}^{\mathscr{D}}(\{x\})$.

$(2) \Rightarrow(1)$ Assume that $x$ and $y$ are any two points of $X$ such that $\mathscr{C}_{\alpha}^{\mathscr{D}}(\{x\}) \cap \mathscr{C}_{\alpha}^{\mathscr{D}}(\{y\}) \neq \phi$. Let $z \in \mathscr{C}_{\alpha}^{\mathscr{D}}(\{x\}) \cap C_{\alpha}^{\mathscr{D}}(\{y\})$. So there exists a net $\left\{x_{\alpha}\right\}_{\alpha \in \Lambda}$ in $\mathscr{C}_{\alpha}^{\mathscr{D}}(\{x\})$ such that $\left\{x_{\alpha}\right\}_{\alpha \in \Lambda} \mathscr{D} \alpha$-converges to $z$. Since $z \in \mathscr{C}_{\alpha}^{\mathscr{D}}(\{y\})$, we have $\left\{x_{\alpha}\right\}_{\alpha \in \Lambda} \mathscr{D} \alpha$-converges to $y$. It follows that $y \in \mathscr{C}_{\alpha}^{\mathscr{D}}(\{x\})$. Similarly, we obtain $x \in \mathscr{C}_{\alpha}^{\mathscr{D}}(\{y\})$. Therefore, $\mathscr{C}_{\alpha}^{\mathscr{D}}(\{x\})=\mathscr{C}_{\alpha}^{\mathscr{D}}(\{y\})$ and, by Theorem $15,(X, \tau)$ is a $\mathscr{D} \alpha-R_{0}$ space.
Definition 13. A topological space $(X, \tau)$ is said to be $\mathscr{D} \alpha-$ $R_{1}$ space if, for $x, y$ in $X$ with $\mathscr{C}_{\alpha}^{\mathscr{D}}(\{x\}) \neq \mathscr{C}_{\alpha}^{\mathscr{D}}(\{y\})$, there exist disjoint $\mathscr{D} \alpha$-open sets $U$ and $V$ such that $\mathscr{C}_{\alpha}^{\mathscr{D}}(\{x\})$ is a subset of $U$ and $\mathscr{C}_{\alpha}^{\mathscr{D}}(\{y\})$ is a subset of $V$.

Theorem 19. Let $(X, \tau)$ be a topological space. Then,

(1) every $\alpha-R_{1}$ space is $\mathscr{D} \alpha-R_{1}$

(2) every $g-R_{1}$ space is $\mathscr{D} \alpha-R_{1}$

Proof. It is obvious.

From the above discussions, we have the following diagram in which the opposite of implications need not be true.

$$
\alpha-R_{1} \longrightarrow \mathscr{D} \alpha-R_{1} \longleftarrow g-R_{1}
$$

Theorem 20. If $(X, \tau)$ is a $\mathscr{D} \alpha-R_{1}$ space, then $(X, \tau)$ is a D $\alpha-R_{0}$ space.

Proof. Let $U$ be $\mathscr{D} \alpha$-open such that $x \in U$. If $y \notin U$, then since $x \notin \mathscr{C}_{\alpha}^{\mathscr{D}}(\{y\}), \mathscr{C}_{\alpha}^{\mathscr{D}}(\{x\}) \neq \mathscr{C}_{\alpha}^{\mathscr{D}}(\{y\})$. Hence, there exists $\mathscr{D} \alpha$-open $V_{y}$ such that $\mathscr{C}_{\alpha}^{\mathscr{D}}(\{y\}) \subset V_{y}$ and $x \notin V_{y}$, which implies that $y \notin \mathscr{C}_{\alpha}^{\mathscr{D}}(\{x\})$. Thus, $\mathscr{C}_{\alpha}^{\mathscr{D}}(\{x\}) \subset U$. Therefore, $(X, \tau)$ is a $\mathscr{D} \alpha-R_{0}$ space.

Theorem 21. A topological space $(X, \tau)$ is said to be a $\mathscr{D} \alpha-$ $R_{1}$ space if and only if $x, y \in X, \operatorname{Ker}_{\alpha}^{\mathscr{D}}(\{x\}) \neq \operatorname{Ker}_{\alpha}^{\mathscr{D}}(\{y\})$, and there exist disjoint $\mathscr{D} \alpha$-open sets $U$ and $V$ such that $\mathscr{C}_{\alpha}^{\mathscr{D}}(\{x\}) \subset U$ and $\mathscr{C}_{\alpha}^{\mathscr{D}}(\{y\}) \subset V$.

Proof. It follows from Lemma 4 (1).

\section{Weakly $\mathscr{D} \boldsymbol{\alpha}-R_{0}$ Space}

Definition 14. A topological space $(X, \tau)$ is said to be weakly $\mathscr{D} \alpha-R_{0}$ space if $\cap_{x \in X} \mathscr{C}_{\alpha}^{\mathscr{D}}(\{x\})=\phi$.

Theorem 22. A topological space $(X, \tau)$ is weakly $\mathscr{D} \alpha-R_{0}$ space if and only if $\operatorname{Ker}_{\alpha}^{\mathscr{D}}(\{x\}) \neq X$ for every $x \in X$.

Proof. Assume that the space $(X, \tau)$ is weakly $\mathscr{D} \alpha-R_{0}$ space. Suppose that there is a point $y$ in $X$ such that $\operatorname{Ker}_{\alpha}^{D}(\{y\})=X$. Then $y \notin O$, where $O$ is some proper $\mathscr{D} \alpha$-open subset of $X$. This implies that $y \in \cap_{x \in X} \mathscr{C}_{\alpha}^{\mathscr{D}}(\{x\})$. But this is a contradiction. Now suppose that $\operatorname{Ker}_{\alpha}^{\mathscr{D}}(\{x\}) \neq X$ for every $x \in X$. If there exists a point $y \in X$ such that $y \in \cap_{x \in X} \mathscr{C}_{\alpha}^{\mathscr{D}}(\{x\})$, then every $\mathscr{D} \alpha$-open set containing $y$ must contain every point of $X$. This implies that the space $X$ is the unique $\mathscr{D} \alpha$-open set containing $y$. Thus, $\operatorname{Ker}_{\alpha}^{\mathscr{D}}(\{x\})=X$, which is a contradiction. Hence, $(X, \tau)$ is a weakly $\mathscr{D} \alpha-R_{0}$ space.

Theorem 23. A topological space $(X, \tau)$ is a weakly $\mathscr{D} \alpha-R_{0}$ space if and only if $\operatorname{Ker}_{\alpha}^{D}(\{x\}) \neq X$ for every $x \in X$. 
Definition 15. A function $f: X \longrightarrow Y$ is said to be always $\mathscr{D} \alpha$-closed if the image of every $\mathscr{D} \alpha$-closed subset of $X$ is $\mathscr{D} \alpha$-closed in $Y$.

Theorem 24. If $f: X \longrightarrow Y$ is an always injective $\mathscr{D} \alpha$-closed function and $X$ is a weakly $\mathscr{D} \alpha-R_{0}$ space, then $Y$ is a weakly $\mathscr{D} \alpha-R_{0}$ space.

Proof. The proof is clear.

Theorem 25. If the topological space $X$ is weakly $\mathscr{D} \alpha-R_{0}$ and $Y$ is any topological space, then the product $X \times Y$ is weakly $\mathscr{D} \alpha-R_{0}$.

Proof. If we show that $\cap{ }_{(x, y) \in X \times Y} \mathscr{C}_{\alpha}^{\mathscr{D}}(\{x, y\})=\phi$, then we are done. Observe that $\cap(x, y) \in X \times Y \mathscr{C}_{\alpha}^{\mathscr{D}}(\{x, y\}) \subset \cap_{(x, y) \in X \times Y}$ $\left(\mathscr{C}_{\alpha}^{\mathscr{D}}(\{x\}) \times \mathscr{C}_{\alpha}^{\mathscr{D}}(\{y\})\right)=\cap_{x \in X} \mathscr{C}_{\alpha}^{\mathscr{D}}(\{x\}) \times \cap_{y \in Y} \mathscr{C}_{\alpha}^{\mathscr{D}}(\{y\})$

$\subset \phi \times Y=\phi$ and hence the proof is completed.

\section{Data Availability}

No data were used to support this study.

\section{Conflicts of Interest}

The authors declare that they have no conflicts of interest.

\section{Acknowledgments}

This work was supported by the Yulin University Industry Collaboration Project (2019-75-3).

\section{References}

[1] O. Njåstad, "On some classes of nearly open sets," Pacific Journal of Mathematics, vol. 15, pp. 961-970, 1965.

[2] D. Andrijevic, "Some properties of the topology of $\alpha$-sets," Matematički Vesnik, vol. 36, pp. 1-10, 1984.

[3] M. Caldas and J. Dontchev, "On spaces with hereditarily compact $\alpha$-topologies," Acta Mathematica Hungarica, vol. 82, pp. 121-129, 1999.

[4] M. Caldas and S. Jafari, "On some low separation axioms in topological spaces," Houston Journal of Mathematics, vol. 29, pp. 93-104, 2003.

[5] M. Caldas and G. Navalagi, "On weakly $\alpha$-open functions between topological spaces," International Journal of Mathematics and Mathematical Sciences, vol. 39, pp. 39-51, 2004.

[6] S. Jafari and T. Noiri, "Some remarks on weakly $\alpha$-continuity," Far East Journal of Mathematical Sciences, vol. 6, pp. 619-625, 1998.

[7] S. N. Maheshwari and S. S. Thakur, "On $\alpha$-irresolute mappings," Tamkang Journal of Mathematics, vol. 11, pp. 209-214, 1980.

[8] H. Maki, R. Devi, and K. Balachandran, " $\alpha$ Generalized-closed sets in topology," Fukuoka University of Education, vol. 42, pp. 13-21, 1993.

[9] T. Noiri, "Weakly $\alpha$-continuous functions," International Journal of Mathematics and Mathematical Sciences, vol. 10, no. 3, pp. 483-490, 1987.

[10] N. Levine, "Generalized closed sets in topology," Rendiconti del Circolo Matematico di Palermo, vol. 19, no. 1, pp. 89-96, 1970.
[11] P. Agashe and N. Levine, "Adjacent topologies," Journal of Mathematics, Tokushima University, vol. 7, pp. 21-35, 1973.

[12] W. Dunham, "T $T_{1 / 2}$-spaces," Kyungpook Mathematical Journal, vol. 17, pp. 161-169, 1977.

[13] W. Dunham and N. Levine, "Further results on generalized closed sets in topology," Kyungpook Mathematical Journal, vol. 20, pp. 169-175, 1980.

[14] W. Dunham, "A new closure operator for non- $T_{1}$ topologies," Kyungpook Mathematical Journal, vol. 22, pp. 55-60, 1982.

[15] O. R. Sayed and A. M. Khalil, "Some applications of D $\alpha$-closed sets in topological spaces," Egyptian Journal of Basic and Applied Sciences, vol. 3, no. 1, pp. 26-34, 2016.

[16] L. Li and Q. Li, "On enriched L-topologies: base and subbase," Journal of Intelligent \& Fuzzy Systems, vol. 28, no. 6, pp. 2423-2432, 2015.

[17] L. Zhou and J. Zhang, "Lukasiewicz semantic MV-topology for MV-algebra and its application to Lukasiewicz propositional logic," Journal of Intelligent \& Fuzzy Systems, vol. 33, no. 1, pp. 377-387, 2017.

[18] M. El-Dardery, A. A. Ramadan, and Y. C. Kim, "L-fuzzy topogenous orders and L-fuzzy topologies," Journal of Intelligent \& Fuzzy Systems, vol. 24, no. 4, pp. 685-691, 2013.

[19] A. Robert and S. P. Missier, “* On semi-closed sets," The Asian Journal of Mathematics, vol. 4, pp. 173-176, 2012.

[20] M. Caldas, D. N. Georgiou, and S. Jafari, "Characterizations of low separation axioms via-open sets and $\alpha \alpha$-closure operator," Bulletin of Parana's Mathematical Society, vol. 21, no. 1-2, pp. 1-14, 2003.

[21] B. Roy, "On generalized R 0 and R 1 spaces," Acta Mathematica Hungarica, vol. 127, no. 3, pp. 291-300, 2010.

[22] M. Caldas, "A note on some applications of $\alpha$-open sets," International Journal of Mathematics and Mathematical Sciences, vol. 2003, pp. 125-130, 2003. 